\title{
APLICAÇÃO DE RESÍDUO DE RECICLAGEM DE PAPEL EM CAMBISSOLO HÁPLICO E SEU EFEITO NO SOLO E NO CULTIVO DE PLANTAS ${ }^{(1)}$
}

\author{
Alvadi Antonio Balbinot Junior ${ }^{(2)}$, Milton da Veiga ${ }^{(3)}$, José Alfredo da Fonseca ${ }^{(4)}$, Gilcimar \\ Adriano Vogt ${ }^{(4)}$, Jackson Adriano Albuquerque ${ }^{(5)}$ \& Epitágoras Rodson Oliveira Costa ${ }^{(6)}$
}

\section{RESUMO}

O processo de reciclagem de papel gera grande quantidade de resíduo e a sua aplicação no solo pode ser uma das estratégias de disposição desse produto, sendo necessário estudar os seus efeitos no solo e nas culturas agrícolas. $O$ objetivo deste trabalho foi avaliar o efeito da aplicação de resíduo de reciclagem de papel sobre atributos químicos de um Cambissolo Háplico muito ácido e na produtividade de grãos de soja e feijão. Utilizou-se o delineamento experimental de blocos completos casualizados, com três repetições. Os tratamentos foram: testemunha, sem calcário ou resíduo; resíduo da reciclagem de papel nas doses de 50, 100, 150, 250, 400 e $600 \mathrm{Mg} \mathrm{ha}^{-1}$, em base úmida; e calcário dolomítico para elevar o pH do solo em água para 6,0. O resíduo corrigiu a acidez do solo, bem como aumentou os teores de cálcio e fósforo. As doses de resíduo não interferiram nos teores de metais pesados no solo e nos grãos. As máximas produtividades de soja e feijão são obtidas nas doses de 323 e $372 \mathrm{Mg} \mathrm{ha}^{-1}$ de resíduo úmido, respectivamente.

Termos de indexação: acidez do solo, metais pesados, produtividade de grãos, Glycine max L., Phaseolus vulgaris L.

(1) Recebido para publicação em 11 de março de 2013 e aprovado em 11 de outubro de 2013.

(2) Pesquisador da Empresa Brasileira de Pesquisa Agropecuária, Embrapa Soja. Rodovia Carlos João Strass, distrito de Warta. Caixa Postal 231. CEP 86001-970 Londrina (PR). E-mail: alvadi.balbinot@embrapa.br

(3) Pesquisador da Empresa de Pesquisa Agropecuária e Extensão Rural de Santa Catarina, Estação Experimental de Campos Novos. BR 282, km 342, Trevo. Caixa Postal 116. CEP 89620-000 Campos Novos (SC). E-mail: milveiga@epagri.sc.gov.br

(4) Pesquisadores da Empresa de Pesquisa Agropecuária e Extensão Rural de Santa Catarina, Estação Experimental de Canoinhas. BR 280, km 219,5, Campo da Água Verde. Caixa Postal 216. CEP 89460-00 Canoinhas (SC). E-mail: fonseca@epagri.sc.gov.br, gilcimar@epagri.sc.gov.br

(5) Professor do Departamento de Solos da Universidade do Estado de Santa Catarina. Av. Luis de Camões, 2090, Conta Dinheiro. CEP 88520-000 Lages (SC). E-mail: jackson.irai@gmail.com

(6) Gerente Florestal da Companhia Volta Grande de Papel. Rua Visconde de Mauá, 366. CEP 89295-000 Rio Negrinho (SC). Email: epitagorasc@cvg.ind.br 


\title{
SUMMARY: PAPER-RECYCLING WASTE APPLICATION TO AN INCEPTISOL AND ITS EFFECT ON SOIL AND CROP CULTIVATION
}

\begin{abstract}
The paper recycling process produces a large amount of waste and applying it on the soil may be one important strategy for use of this product. However, it is necessary to study the effects of application of this waste product on soil properties and crop performance. The aim of this study was to evaluate the effect of paper recycling waste on the chemical properties of an acidic Inceptisol and on the yield of soybean and common bean. A randomized complete block design was used, with three replications. The following treatments were evaluated: paper recycling waste at the rates of 50, 100, 150, 250, 400 and $600 \mathrm{Mg} \mathrm{ha}^{-1}$ of wet mass; dolomitic limestone to raise soil $\mathrm{pH}$ in water to 6.0; and a control, without waste or limestone application. The recycling waste increased soil $p H$ and Ca and P levels. The application rates of recycling waste did not affect the levels of heavy metals in the soil and in the grains, even at the higher rates. The greatest yields of soybean and common bean were obtained at the rates of 323 and $372 \mathrm{Mg} \mathrm{ha}^{-1}$ of waste wet mass, respectively.
\end{abstract}

Index terms: soil acidity, heavy metals, grain yield, Glycine max L., Phaseolus vulgaris L..

\section{INTRODUÇÃO}

A grande quantidade de resíduos agrícolas, urbanos e industriais gerados nas diversas regiões do país tem estimulado o estudo de formas para a sua disposição, que resultem em baixo potencial de contaminação ambiental. O uso desses materiais na agricultura, em substituição parcial ou total de corretivos ou fertilizantes minerais, tem apresentado resultados promissores em diferentes condições (Nkana et al., 1999; Mäkela et al., 2012). As escórias de siderurgia, por exemplo, podem ser utilizadas como corretivo da acidez do solo (Nogueira et al., 2012), potencial que também apresenta o resíduo de processamento de bauxita (Nobile et al., 2011) e o lodo de curtume (Gianello et al., 2011). No entanto, alguns desses materiais contêm alta concentração de metais pesados, destacando-se o $\mathrm{Cr}$, nos resíduos de curtume (Gianello et al., 2011); o Cu e o Zn, nos dejetos líquidos de suínos (Veiga et al., 2012); e vários elementos no lodo de estações de tratamento de esgoto (Borges \& Coutinho, 2004; Rangel et al., 2006). Esses elementos podem se acumular nas camadas superficiais do solo e serem perdidos por escoamento superficial (Girotto et al., 2010) ou, ainda, absorvidos pelas culturas (Borges \& Coutinho, 2004; Rangel et al., 2006; Gianello et al., 2011). O aumento na absorção e no acúmulo de elementos potencialmente tóxicos nas folhas e nos grãos das culturas, mesmo que na maioria das vezes não atinja os teores máximos permitidos em alimentos, resulta em risco de sua transferência na cadeia alimentar (Rangel et al., 2006), com impacto sobre a fauna e a flora do solo e mesmo sobre os animais que consomem essa biomassa. Alguns estudos avaliam a aplicação de resíduos da indústria de papel, que possuem potencial para corrigir a acidez de solos cultivados com culturas agrícolas e florestais (Phillips et al., 1997; Feldkirchner et al., 2003; Nunes et al., 2008; Ríos et al., 2012).

Nas últimas décadas, houve um processo de consolidação das indústrias que utilizam papel descartado na produção de novos produtos. A transformação de rejeitos de papel, como revistas e jornais, em novos produtos de valor comercial, como papel higiênico, gera quantidade expressiva de resíduo, o qual apresenta característica de uma massa fibrosa de cor acinzentada, sendo classificado como um resíduo IIA - não inerte (ABNT, 2004). Em razão do elevado volume produzido e da baixa permeabilidade do resíduo, sua alocação em aterros sanitários tem custo elevado, além de representar um passivo ambiental.

No resíduo gerado pelo processo de reciclagem de papel, constata-se a presença de compostos que podem atuar como corretivos da acidez do solo, principalmente óxidos de $\mathrm{Ca}$ e $\mathrm{Mg}$ (Balbinot Junior et al., 2006a). O emprego desses resíduos para correção da acidez do solo, em doses apropriadas, tem se mostrado eficaz (Balbinot Junior et al., 2006b; Costa et al., 2009; Balbinot Junior et al., 2010a). Todavia, há carência de resultados de pesquisas sobre os efeitos da aplicação desse resíduo nos atributos químicos de solos rasos e muito ácidos e na produtividade de culturas.

A hipótese deste trabalho é de que a aplicação de resíduo de reciclagem de papel em um Cambissolo Háplico muito ácido aumenta o $\mathrm{pH}$ e os teores de Ca e Mg no solo, bem como a produtividade das culturas, sem alterar significativamente os teores de metais pesados no solo e nos grãos. Nesta pesquisa, objetivouse avaliar o efeito de resíduo de reciclagem de papel, aplicado em diferentes doses, nos atributos químicos de um Cambissolo Háplico muito ácido e na produtividade das culturas de soja e feijão.

\section{MATERIAL E MÉTODOS}

O experimento foi implantado no município de Canoinhas, SC (longitude $50^{\circ} 16^{\prime}$ oeste, latitude $26^{\circ} 22 \mathrm{sul}$ e altitude de $800 \mathrm{~m}$ ), em um Cambissolo Háplico muito ácido (Embrapa, 2004). Antes da aplicação dos tratamentos, o solo apresentava os seguintes atributos: $682 \mathrm{~g} \mathrm{~kg}^{-1}$ de argila; $\mathrm{pH}\left(\mathrm{H}_{2} \mathrm{O}\right)$ $=4,7 ;$ índice $\mathrm{SMP}=4,6 ; \mathrm{P}$ (resina) $=15 \mathrm{mg} \mathrm{dm}^{-3}$; 
$\mathrm{K}=184 \mathrm{mg} \mathrm{dm}^{-3} ; \mathrm{MO}=46 \mathrm{~g} \mathrm{~kg}^{-1} ; \mathrm{Al}=8,6 \mathrm{cmol}_{\mathrm{c}} \mathrm{dm}^{-3} ;$ $\mathrm{Ca}=3,8 \mathrm{cmol}_{\mathrm{c}} \mathrm{dm}^{-3} ; \mathrm{Mg}=0,5 \mathrm{cmol}_{\mathrm{c}} \mathrm{dm}^{-3} ;$ e CTC $=$ $24,9 \mathrm{cmol}_{\mathrm{c}} \mathrm{dm}^{-3}$.

Os tratamentos consistiram da aplicação das doses de 50,100, 150, 250, 400 e $600 \mathrm{Mg} \mathrm{ha}^{-1}$ de resíduo de reciclagem de papel (base úmida), as quais foram determinadas com base no trabalho desenvolvido por Balbinot Junior et al. (2006a,b). Também foi utilizado um tratamento referência, com aplicação de 15,1 $\mathrm{Mg} \mathrm{ha}^{-1}$ de calcário dolomítico (PRNT de $100 \%$ ), para atingir pH 6,0 (CQFSRS/SC, 2004), e um tratamento testemunha, sem aplicação de resíduo ou calcário. O delineamento experimental foi o de blocos completos casualizados, com três repetições, com unidades experimentais de $40 \mathrm{~m}^{2}$ (5 x $\left.8 \mathrm{~m}\right)$.

Algumas características químicas do resíduo, determinadas segundo métodos descritos por Tedesco et al. (1995), são apresentadas no quadro 1. O resíduo de reciclagem de papel e o calcário foram distribuídos nas parcelas em agosto de 2008, sendo imediatamente incorporados ao solo, até a

Quadro 1. Umidade gravimétrica e caracterização química do resíduo de reciclagem de papel utilizados no experimento

\begin{tabular}{|c|c|}
\hline Característica $^{(1)}$ & Valor \\
\hline Umidade $\left(\mathrm{g} \mathrm{kg}^{-1}\right)$ & 630 \\
\hline $\mathrm{pH}$ & 7,7 \\
\hline Carbono orgânico $\left(\mathrm{g} \mathrm{kg}^{-1}\right)$ & 200 \\
\hline Nitrogênio (TKN) $\left(\mathrm{g} \mathrm{kg}^{-1}\right)$ & 1,7 \\
\hline $\mathrm{P}_{2} \mathrm{O}_{5}$ total $\left(\mathrm{g} \mathrm{kg}^{-1}\right)$ & 0,7 \\
\hline $\mathrm{K}_{2} \mathrm{O}$ total $\left(\mathrm{g} \mathrm{kg}^{-1}\right)$ & 0,5 \\
\hline $\mathrm{CaO}$ total $\left(\mathrm{g} \mathrm{kg}^{-1}\right)$ & 240 \\
\hline $\mathrm{MgO}$ total $\left(\mathrm{g} \mathrm{kg}^{-1}\right)$ & 3,0 \\
\hline $\mathrm{S}$ total $\left(\mathrm{g} \mathrm{kg}^{-1}\right)$ & 0,7 \\
\hline $\mathrm{Cu}$ total $\left(\mathrm{mg} \mathrm{kg}^{-1}\right)$ & 39 \\
\hline Zn total $\left(\mathrm{mg} \mathrm{kg}^{-1}\right)$ & 306 \\
\hline Ferro total $\left(\mathrm{g} \mathrm{kg}^{-1}\right)$ & 2,4 \\
\hline Mn total $\left(\mathrm{mg} \mathrm{kg}^{-1}\right)$ & 40 \\
\hline $\mathrm{Na}$ total $\left(\mathrm{g} \mathrm{kg}^{-1}\right)$ & 2,3 \\
\hline B total $\left(\mathrm{mg} \mathrm{kg}^{-1}\right)$ & $<1$ \\
\hline $\mathrm{Pb}$ total $\left(\mathrm{mg} \mathrm{kg}^{-1}\right)$ & 7 \\
\hline Ni total $\left(\mathrm{mg} \mathrm{kg}^{-1}\right)$ & 4 \\
\hline $\mathrm{Cd}$ total $\left(\mathrm{mg} \mathrm{kg}^{-1}\right)$ & $<0,2$ \\
\hline $\mathrm{Cr}$ total $\left(\mathrm{mg} \mathrm{kg}^{-1}\right)$ & 4 \\
\hline $\mathrm{Hg}\left(\mathrm{mg} \mathrm{kg}{ }^{-1}\right)$ & 0,1 \\
\hline Mo (mg kg-1) & 3 \\
\hline Co total $\left(\mathrm{mg} \mathrm{kg}^{-1}\right)$ & 5 \\
\hline $\mathrm{Al}$ total $\left(\mathrm{g} \mathrm{kg}^{-1}\right)$ & 25 \\
\hline Cinzas $\left(\mathrm{g} \mathrm{kg}^{-1}\right)$ & 610 \\
\hline Cond. elétrica $\left(\mathrm{dS} \mathrm{cm}^{-1}\right)$ & 0,9 \\
\hline Poder de neutralização (\%) & 44 \\
\hline
\end{tabular}

(1) Resultados expressos no material seco a $75{ }^{\circ} \mathrm{C}$ (Tedesco et al., 1995). Média de três repetições. profundidade de $20 \mathrm{~cm}$, por meio de aração realizada com arado de discos e posteriormente três passagens de grade niveladora.

$\mathrm{Na}$ safra 2008/09, foi cultivado milho (dados publicados em De Marco et al., 2012). Nas safras 2009/ 10 e 2010/11, cultivaram-se, respectivamente, soja (cultivar CD 206 em espaçamento de $0,45 \mathrm{~m}$ e 220 mil plantas ha-1) e feijão (cultivar IPR Tiziu em espaçamento de $0,45 \mathrm{~m}$ e 250 mil plantas ha ${ }^{-1}$ ). Nos períodos entre as safras estivais foi cultivado azevém, sem adubação. Todas as culturas foram semeadas no sistema de plantio direto. A adubação de base realizada na soja e no feijão foi a mesma para todos os tratamentos, seguindo a indicação da CQFSRS/SC (2004). Os tratos culturais empregados nas culturas de soja e feijão foram aqueles indicados pela pesquisa. As plantas de soja e feijão presentes na área útil das parcelas $\left(5,4 \mathrm{~m}^{2}\right)$ foram colhidas manualmente, trilhadas, e os grãos, pesados, sendo a produtividade corrigida para $13 \%$ de umidade. Os resíduos das culturas de verão e de inverno foram distribuídos uniformemente nas parcelas, para não haver exportação de nutrientes via palha.

Em abril de 2010 e de 2011, foram coletadas, nas entrelinhas das culturas de soja e feijão, 15 subamostras de solo em cada unidade experimental, na camada de $0-20 \mathrm{~cm}$, as quais formaram uma amostra composta. Nessas amostras, foram determinados: $\mathrm{pH}\left(\mathrm{H}_{2} \mathrm{O}\right)$; teores de $\mathrm{Ca}, \mathrm{Mg}$ e $\mathrm{Na}$ trocáveis, extraídos com $\mathrm{KCl} 1 \mathrm{~mol} \mathrm{~L}^{-1}$; $\mathrm{K}$ e P, por Mehlich-1 (Tedesco et al., 1995); Hg (USEPA, 1993, 7471 A); e Pb, Ni, Cd e Cr (USEPA, 1993, 3050). O método USEPA 3050 extrai os metais considerados ambientalmente disponíveis. Em cada parcela, coletou-se uma amostra de grãos de soja e feijão para determinação dos teores de $\mathrm{N}$ (Kjeldahl 0,01 \%), P, $\mathrm{K}, \mathrm{Ca}, \mathrm{Mg}, \mathrm{S}, \mathrm{Cu}, \mathrm{Zn}, \mathrm{Fe}, \mathrm{Mn}, \mathrm{Na}, \mathrm{B}, \mathrm{Cd}, \mathrm{Cr}, \mathrm{Ni}$ e Pb, determinados por espectrometria de emissão ótica, em plasma indutivamente acoplado (ICP-OES) e $\mathrm{Hg}$ (USEPA, 1993, 7471).

Os dados foram analisados estatisticamente por meio de análise de variância e teste F. Quando identificada a existência de diferença significativa $(p<0,05)$ entre tratamentos, realizou-se análise de regressão. Selecionaram-se o modelo que apresentou o melhor ajuste aos dados e ao fenômeno investigado e os coeficientes significativos a $5 \%$.

\section{RESULTADOS E DISCUSSÃO}

O resíduo de reciclagem de papel aumentou o $\mathrm{pH}$ $\left(\mathrm{H}_{2} \mathrm{O}\right)$ (Figura 1a,b), o que comprova seu efeito corretivo da acidez do solo, como discutido por Balbinot Jr. et al. (2006a) e Balbinot Jr. et al. (2010a), em trabalhos realizados em um Latossolo Vermelho. Mäkela et al. (2012) também verificaram que a aplicação de resíduo de papel corrigiu a acidez do solo. 

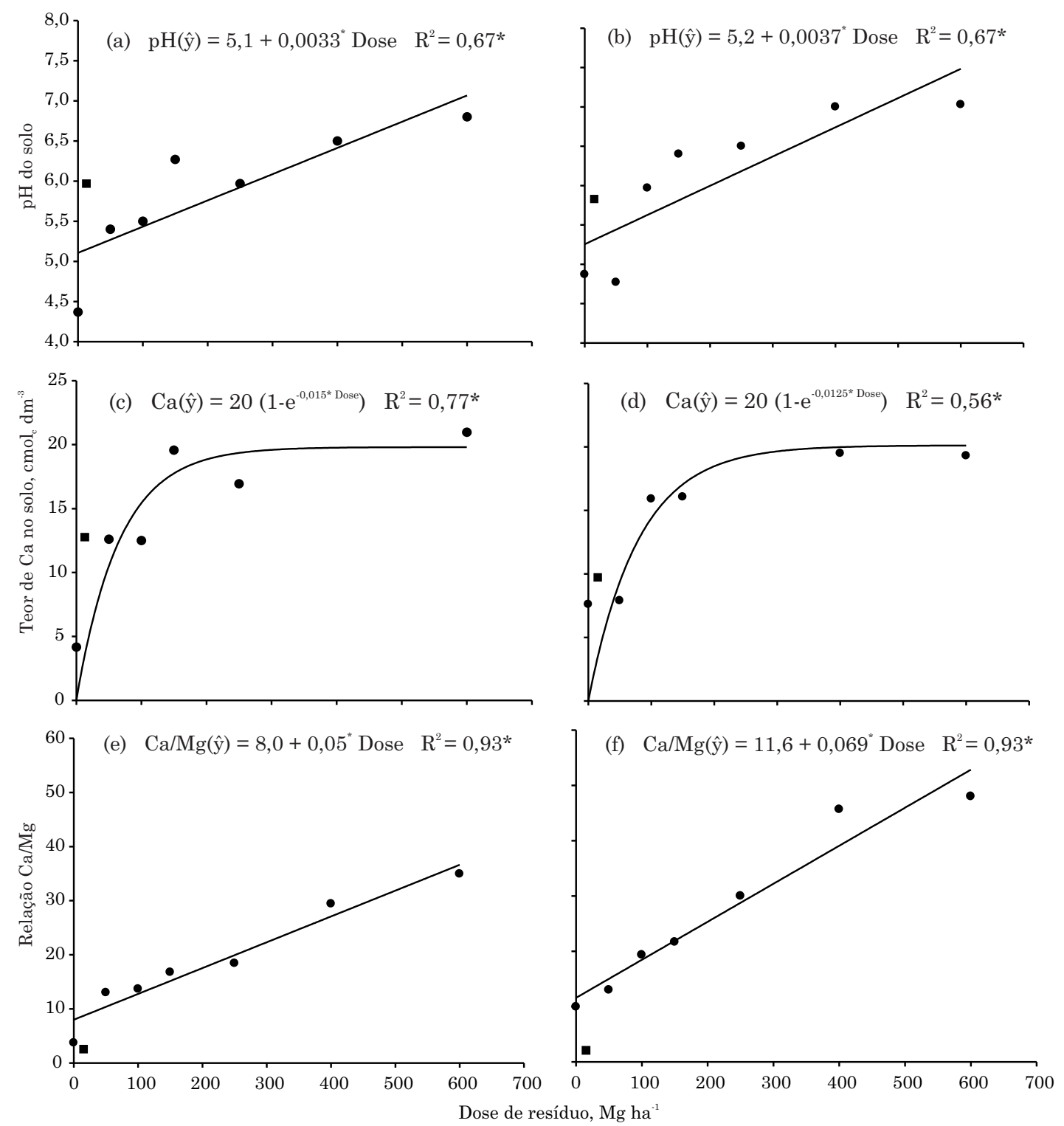

Figura 1. pH do solo determinado em água (a, b), teores de Ca no solo (c, d) e relação $\mathrm{Ca} / \mathrm{Mg}(\mathrm{e}, \mathrm{f})$ em função de doses de resíduo de reciclagem de papel (•) e com aplicação de calcário (ロ) (amostragens: abril de 2010, na esquerda, e abril de 2011 , na direita). * Significativo a $5 \%$ pelo teste t.

Aos 20 e 32 meses após a aplicação, respectivamente abril de 2010 e abril de 2011 , o $\mathrm{pH}\left(\mathrm{H}_{2} \mathrm{O}\right)$ foi superior a 5,5 nas doses superiores a $150 \mathrm{Mg} \mathrm{ha}^{-1}$ (Figura 1a,b). $\mathrm{O} \mathrm{pH}\left(\mathrm{H}_{2} \mathrm{O}\right)$ adequado para a maioria das culturas anuais se situa na faixa de 5,5 a 6,5 (Marschner, 1995). Nas doses mais altas, após 32 meses da aplicação, o pH estava próximo de 7,0, o que, segundo KabataPendias \& Pendias (1984), pode reduzir tanto a atividade microbiana como a disponibilidade de alguns micronutrientes, como $\mathrm{Mn}, \mathrm{Fe}, \mathrm{Zn}$ e $\mathrm{Cu}$. Nas coletas realizadas em abril de 2010 e de 2011, o pH $\left(\mathrm{H}_{2} \mathrm{O}\right)$ estava próximo a 6,0 com a aplicação de calcário, conforme calculado na implantação do experimento. As doses de resíduo necessárias para elevar o $\mathrm{pH}$ para 6,0 foram de 273 e $203 \mathrm{Mg} \mathrm{ha}^{-1}$, nas coletas realizadas em abril de 2010 e de 2011, respectivamente.
Observou-se aumento no teor de $\mathrm{Ca}^{2+}$ com as doses de resíduo, até, aproximadamente, $200 \mathrm{Mg} \mathrm{ha}^{-1}$ e no tratamento com calcário (Figura 1c,d). Isso ocorreu porque o resíduo e o calcário testados possuem esse elemento em sua composição. Nkana et al. (1999) também verificaram aumentos significativos nos teores de Ca no solo com a aplicação de resíduo de indústria de papel. Em decorrência do aumento no teor de Ca promovido pela aplicação do resíduo no solo, a relação $\mathrm{Ca} / \mathrm{Mg}$ aumentou linearmente até próximo a 50, aos 32 meses após a aplicação (Figura 1e,f). No entanto, com a aplicação de calcário, houve comportamento inverso, ou seja, redução da relação $\mathrm{Ca} / \mathrm{Mg}$, comparativamente à testemunha. $\mathrm{O}$ aumento da relação $\mathrm{Ca} / \mathrm{Mg}$ pode diminuir a absorção de $\mathrm{K}$ e de Mg e o crescimento das culturas (Medeiros et al., 2008) 
e deve ser considerado quando do uso de resíduos. Em muitas áreas cultivadas, a relação $\mathrm{Ca} / \mathrm{Mg}$ é inferior a 2, em razão da utilização intensa de calcário dolomítico (Oliveira et al., 2002), situação em que o resíduo testado pode ser usado para elevar essa relação, mesmo tendo sido observado que o rendimento da maior parte das culturas não é influenciado por relações $\mathrm{Ca} / \mathrm{Mg}$, variando de 0,5 até mais de 10 , desde que nenhum dos dois nutrientes esteja em deficiência (CQFSRS/SC, 2004). Adicionalmente, esse resíduo pode ser usado em culturas que requerem elevada disponibilidade de $\mathrm{Ca}$ no solo para adequado crescimento e desenvolvimento, como, por exemplo, a maçã e o tomate.

Foi constatado aumento nos teores de P com a elevação das doses de resíduo úmido até 284 e $318 \mathrm{Mg}_{\text {ha }}{ }^{-1}$, aos 20 e 32 meses após a aplicação, respectivamente (Figura 2a,b). Doses acima dessas reduziram o teor de $\mathrm{P}$, provavelmente em razão da formação de fosfato de cálcio. Isso ocorre quando há alta concentração de Ca no solo e pH elevado, conforme relatado por Ernani et al. (2000). Além disso, por causa do aumento de $\mathrm{pH}$ ocasionado pelo resíduo, pode ter ocorrido perda de atividade do extrator na determinação do $\mathrm{P}$ (Novais

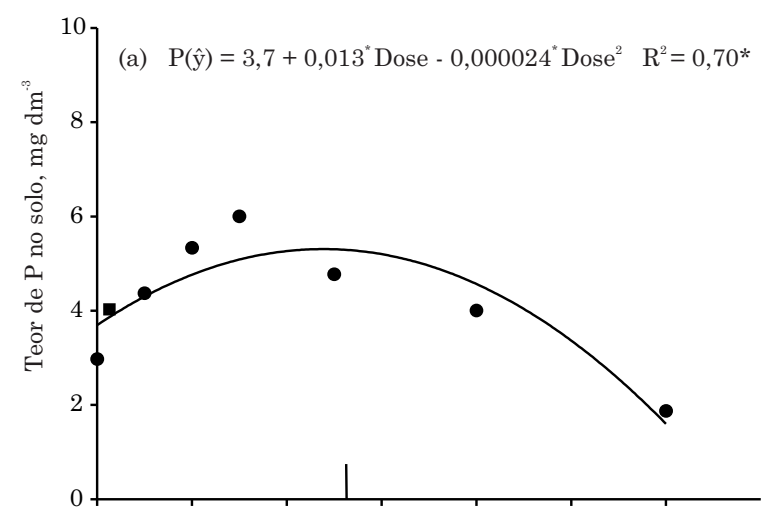

(b) $\mathrm{P}(\hat{\mathrm{y}})=5,0+0,02^{*}$ Dose - 0,000003 ${ }^{*}$ Dose $^{2} \mathrm{R}^{2}=0,55^{*}$
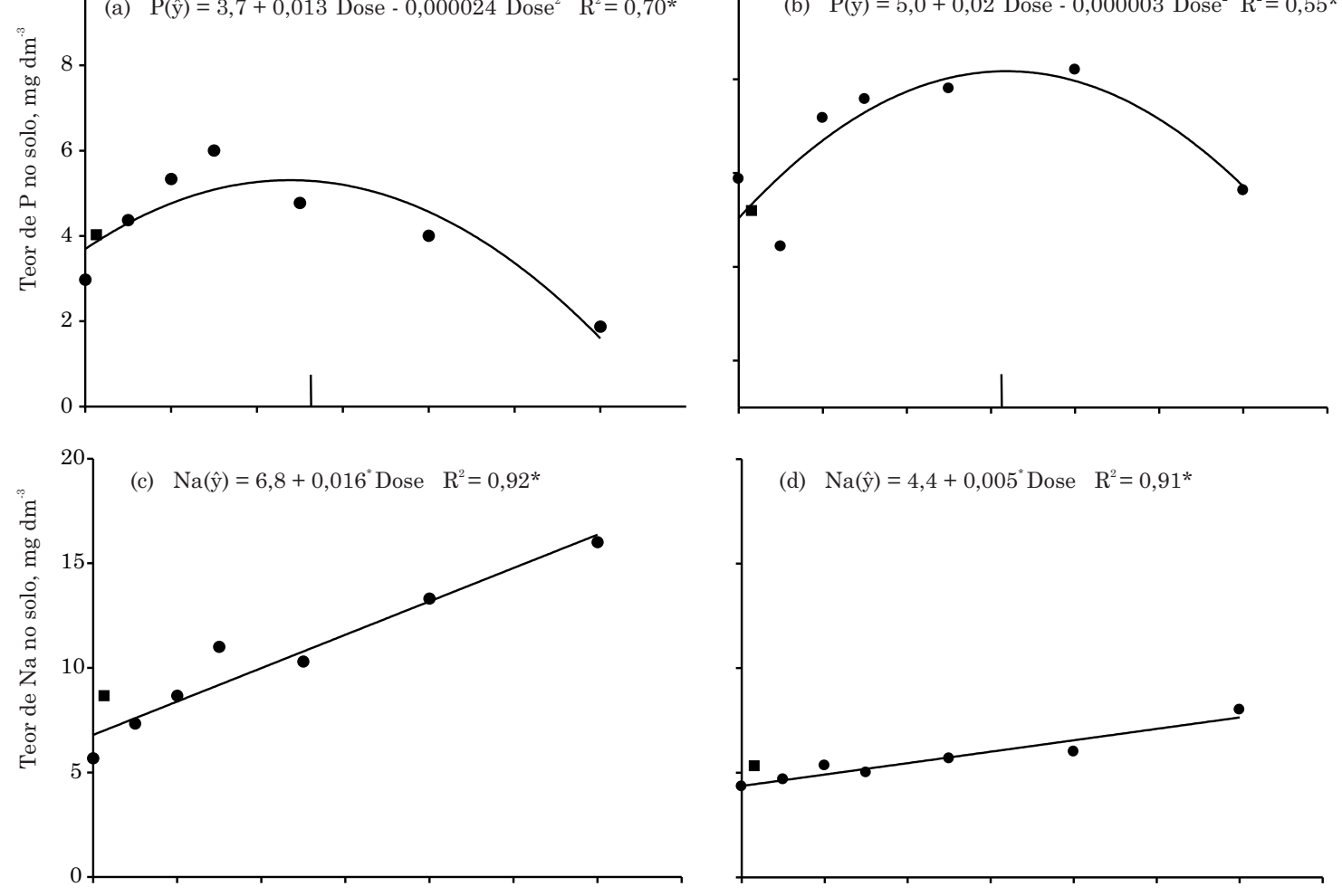

(d) $\mathrm{Na}(\hat{\mathrm{y}})=4,4+0,005^{*}$ Dose $\mathrm{R}^{2}=0,91^{*}$
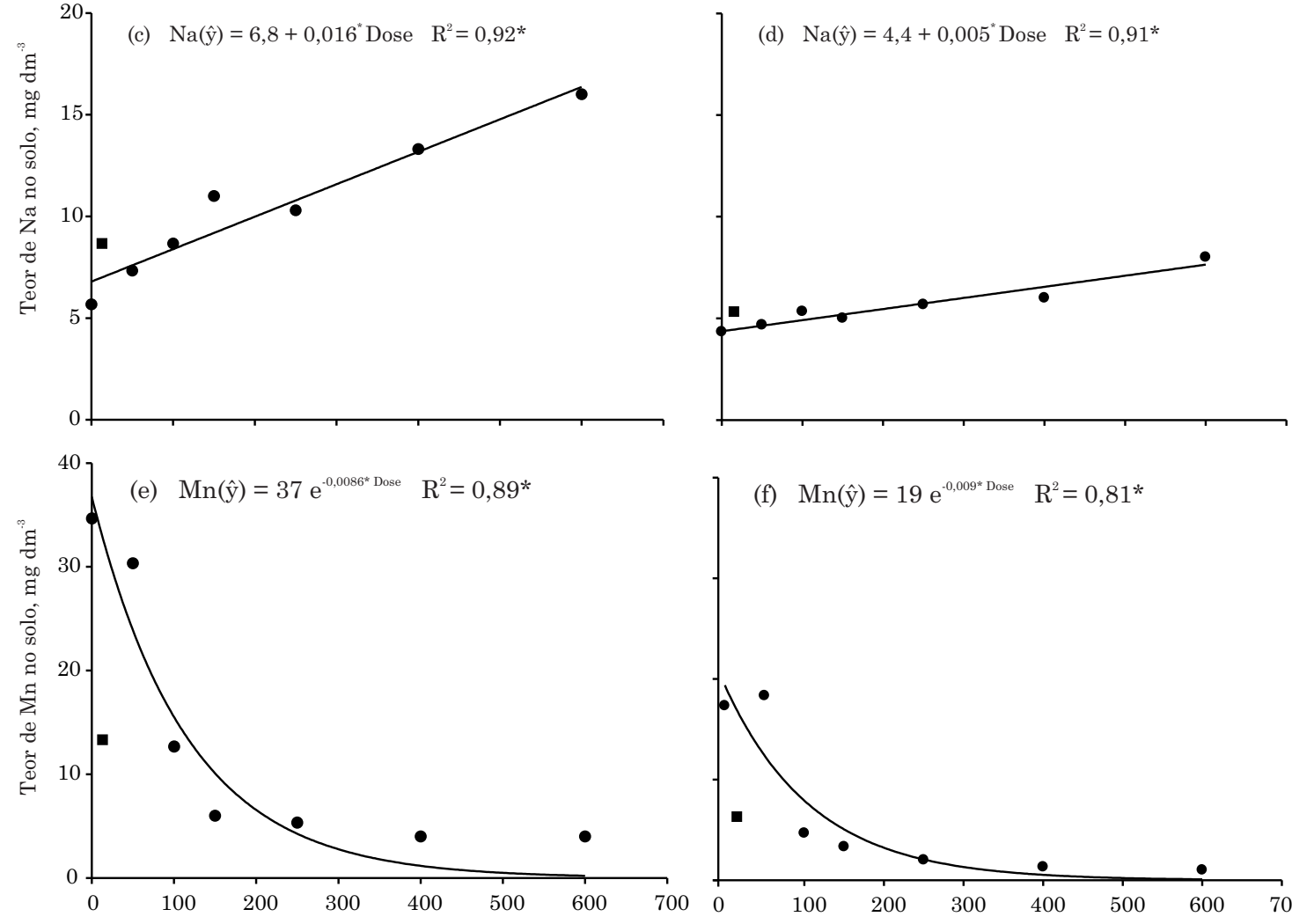

(f) $\operatorname{Mn}(\hat{y})=19 \mathrm{e}^{-0,009^{*} \text { Dose }} \quad \mathrm{R}^{2}=0,81^{*}$

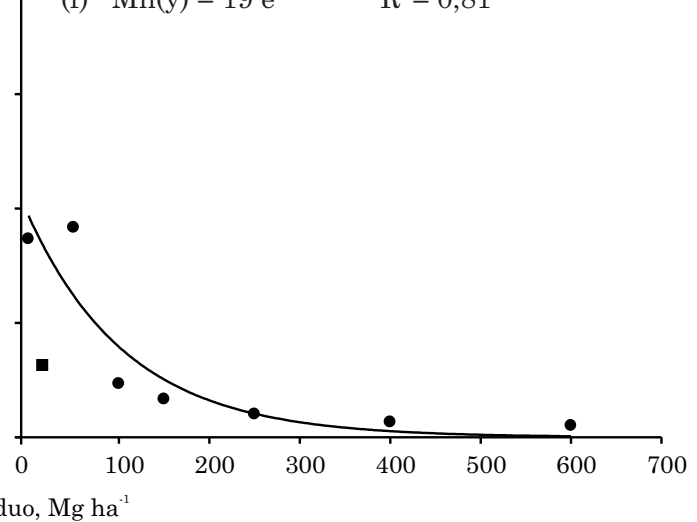

Figura 2. Teores de $\mathrm{P}$ no solo (Mehlich-1) (a, b), Na (c, d) e Mn (e, f) em função de doses de resíduo de reciclagem de papel (•) e com aplicação de calcário ( ( ) (amostragens: abril de 2010, na esquerda, e abril de 2011, na direita). *Significativo a $5 \%$ pelo teste t. (|) máxima eficiência técnica. 
\& Smyth, 1999). Aos 20 meses após a aplicação, na maior dose de resíduo, o teor de $\mathrm{P}$ foi inferior à testemunha, indicando expressiva formação de fosfato de cálcio, o que é indesejável, já que o $\mathrm{P}$ é um macronutriente e os seus teores em solos do sul do Brasil em geral são baixos (CQFSRS/SC, 2004), fato relacionado com a constituição do material de origem e a mineralogia do solo (Almeida et al., 2003). Em trabalho realizado com resíduo similar, em um Latossolo Vermelho, não foi verificada redução do teor de $\mathrm{P}$ em doses superiores a $500 \mathrm{Mg} \mathrm{ha}^{-1}$ de resíduo úmido (Balbinot Jr. et al., 2010a), diferindo do resultado obtido neste trabalho.

Observou-se aumento linear no teor de $\mathrm{Na}$ com o acréscimo da dose de resíduo (Figura 2c,d), pois o resíduo possui $\mathrm{Na}$ em sua composição (Quadro 1). Aumento excessivo de $\mathrm{Na}$ no solo pode ocasionar problemas como salinização e dispersão de argila, comprometendo a estrutura do solo (Medeiros et al., 2009). Contudo, o aumento do teor de Na observado não ultrapassou o teor crítico desse elemento no solo para o desenvolvimento das principais culturas, acima de $15 \%$ da CTC (Fassbender \& Bornemiza, 1994). Esse resultado também foi observado em experimento conduzido em vasos, com um Latossolo Vermelho e resíduo similar (Balbinot Junior et al., 2006a). Na coleta realizada em abril de 2011, os teores foram inferiores à de abril de 2010, possivelmente em razão da lixiviação do $\mathrm{Na}$, já que é um elemento monovalente e com grande raio hidratado, favorecendo sua mobilidade para camadas subsuperficiais.

O teor de Mn no solo diminuiu com a aplicação do resíduo ou calcário (Figura 2e,f). É provável que isso tenha ocorrido, principalmente, em razão do aumento do $\mathrm{pH}$ do solo propiciado pelo resíduo ou calcário. O aumento do $\mathrm{pH}$ reduz a disponibilidade de $\mathrm{Mn}$, principalmente porque diminui a sua solubilidade e aumenta a sua adsorção no solo (Borkert et al., 2001). Teores de Mn abaixo de $2,5 \mathrm{mg} \mathrm{dm}^{-3}$ são considerados baixos (CQFSRS/SC, 2004), conforme observado na amostragem realizada 32 meses após a aplicação para as doses superiores a, aproximadamente, $300 \mathrm{Mg} \mathrm{ha}^{-1}$ de resíduo úmido. Nesse sentido, a redução dos teores de Mn no solo é uma das consequências negativas do uso de altas doses de resíduo de reciclagem de papel.

Verificou-se que a aplicação de resíduo de reciclagem de papel, mesmo nas maiores doses, não alterou estatisticamente os teores de $\mathrm{Hg}, \mathrm{Pb}, \mathrm{Ni}, \mathrm{Cd}$ e Cr disponíveis no solo (Quadro 2), possivelmente em decorrência da baixa concentração desses elementos no resíduo (Quadro 1). A Resolução No 420/2009 do Conama (2009), considerando a necessidade de prevenção da contaminação do solo visando à manutenção de sua funcionalidade e proteção da qualidade das águas superficiais e subterrâneas, lista valores de prevenção (VP) para solos, sendo: 0,5 de $\mathrm{Hg}, 72$ de $\mathrm{Pb}, 30$ de Ni, 1,3 de Cd e 75 de $\mathrm{Cr}$, todos expressos em $\mathrm{mg} \mathrm{kg}^{-1}$. Valor de prevenção é a concentração de valor limite de determinada substância no solo, tal que ele seja capaz de sustentar as suas funções principais (Conama, 2009). Os resultados deste estudo corroboram com os obtidos por Balbinot Junior et al. (2006a) e por Balbinot Junior et al. (2010a), os quais utilizaram resíduo similar em um Latossolo Vermelho. Em relação a outros produtos aplicados no solo, Girotto et al. (2010) verificaram acúmulo de $\mathrm{Cu}$ e Zn na sua camada superficial, com aplicação continuada de dejeto líquido de suínos em um Argissolo. Gianello et al. (2011) detectaram acúmulo de Cr no solo com a aplicação de resíduos de curtume.

Alterações químicas observadas após a adição do resíduo, principalmente com a elevação de $\mathrm{pH}$ e de teores de Ca aumentaram a produtividade de grãos da cultura da soja até a dose de $323 \mathrm{Mg} \mathrm{ha}^{-1}$, que foi o ponto de máxima eficiência técnica, estimado com base na primeira derivada do modelo ajustado (Figura 3a). Para o feijão, a máxima eficiência técnica foi obtida na dose de $372 \mathrm{Mg} \mathrm{ha}^{-1}$ (Figura $3 \mathrm{~b}$ ). As maiores doses testadas reduziram a produtividade das culturas, pois o $\mathrm{pH}$ aumentou acima do recomendado pela CQFSRS/ SC (2004) e, provavelmente pela diminuição da disponibilidade de $\mathrm{P}$ e Mn, apesar de não terem sido observados sintomas visuais de deficiência desses elementos nas plantas, bem como o resíduo aplicado em altas doses pode ter alterado alguns atributos

Quadro 2. Teores de mercúrio $(\mathrm{Hg})$, chumbo $(\mathrm{Pb})$, níquel $(\mathrm{Ni})$, cádmio(Cd) e cromo $(\mathrm{Cr})$ no solo, aos 20 e 32 meses após a aplicação de resíduo de reciclagem de papel e calcário dolomítico (coletas em abril de 2010 e de 2011)

\begin{tabular}{cccccc}
\hline \multirow{2}{*}{ Tratamento } & $\mathbf{H g}^{(1,2)}$ & $\mathbf{P b}^{(\mathbf{1})}$ & $\mathbf{N i}^{(\mathbf{1})}$ & $\mathbf{C d}^{(\mathbf{1})}$ & $\mathbf{C r}^{(\mathbf{1})}$ \\
\cline { 2 - 6 } Mg ha $^{-1}$ & \multicolumn{5}{c}{$\mathrm{mg} \mathrm{kg}^{-1}$} \\
\cline { 2 - 6 } 0 & 0,06 & 20 & 15 & 0,23 & 33 \\
50 & 0,06 & 20 & 17 & 0,20 & 38 \\
100 & 0,06 & 17 & 15 & 0,20 & 31 \\
150 & 0,07 & 18 & 16 & 0,20 & 35 \\
250 & 0,07 & 18 & 14 & 0,20 & 31 \\
400 & 0,06 & 17 & 15 & 0,20 & 32 \\
600 & 0,07 & 21 & 18 & 0,23 & 26 \\
Calcário & 0,06 & 19 & 17 & 0,20 & 36 \\
& & & 2011 & & \\
0 & 0,04 & 24 & 11 & 0,20 & 26 \\
50 & 0,05 & 22 & 12 & 0,20 & 27 \\
100 & 0,04 & 22 & 12 & 0,20 & 27 \\
150 & 0,05 & 25 & 14 & 0,20 & 29 \\
250 & 0,05 & 24 & 13 & 0,20 & 29 \\
400 & 0,05 & 24 & 13 & 0,20 & 30 \\
600 & 0,05 & 24 & 12 & 0,20 & 27 \\
Calcário & 0,05 & 24 & 14 & 0,20 & 29 \\
\hline
\end{tabular}

(1) Resultados expressos no material seco a $45^{\circ} \mathrm{C}$. Média de três repetições. Em cada repetição foram feitas duas determinações. (2) Determinado em vapor frio e outros metais em ICP-OES. 

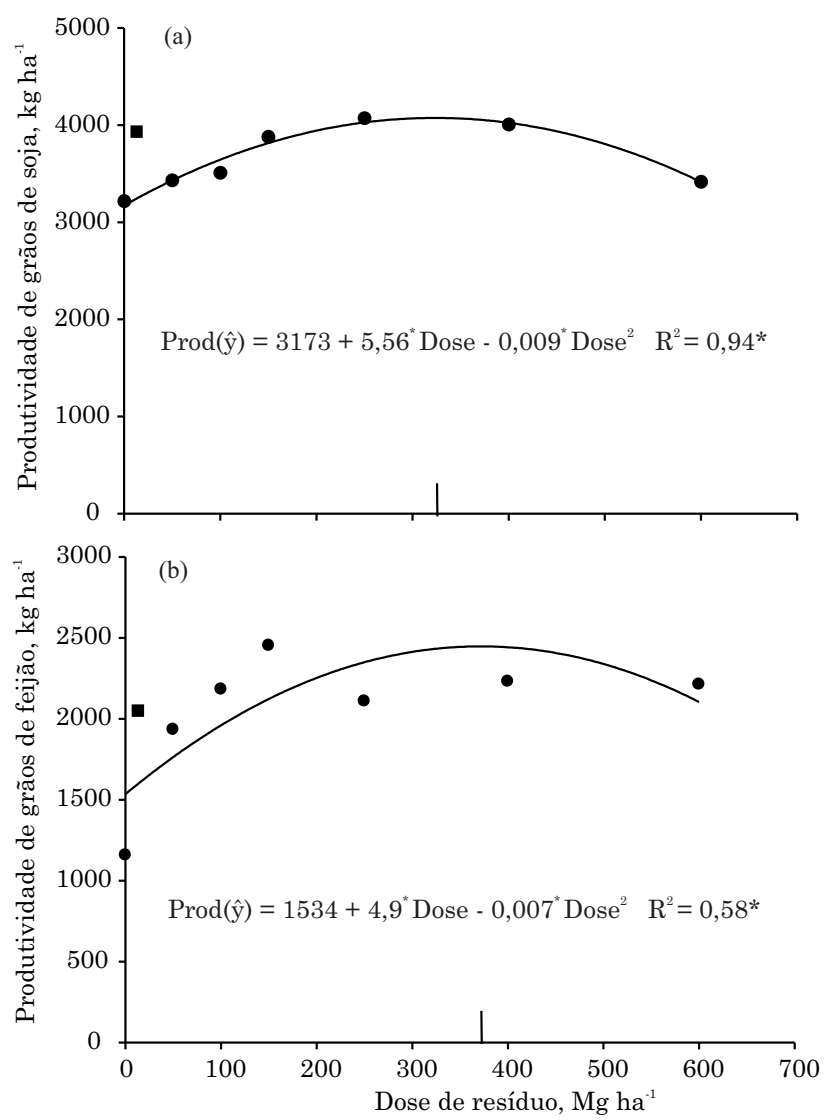

Figura 3. Produtividade de grãos de soja (a) e feijão (b) em função de doses de resíduo de reciclagem de papel (•) e com aplicação de calcário ( $\square$ ). * Significativo a $5 \%$ pelo teste t. (|) máxima eficiência técnica.

físicos do solo, reduzindo o crescimento das raízes e, por consequência, a produtividade de grãos.

Não foram observadas variações nos teores de $\mathrm{Hg}$, $\mathrm{Pb}, \mathrm{Ni}$, Cd e Cr nos grãos de soja e feijão (Quadro 3), concordando com os resultados de Balbinot Junior et al. (2010b), os quais não verificaram efeito da aplicação de resíduo de reciclagem de papel sobre os teores desses metais nos grãos de soja e milho em um Latossolo Vermelho.

Considerando-se os resultados, a hipótese deste trabalho foi comprovada, pois em doses intermediárias de resíduo houve melhoria da fertilidade do solo, o que se refletiu em aumento de produtividade de grãos de soja e feijão em relação à testemunha. Além disso, não houve alterações nos teores ambientalmente disponíveis de metais pesados no solo e nos grãos produzidos, indicando ser possível o uso do resíduo industrial testado como corretivo da acidez do solo e como fonte de cálcio para as culturas. No entanto, salienta-se que há necessidade de estudos adicionais para verificar os efeitos agronômicos e ambientais da aplicação do resíduo em questão, sobretudo considerando reaplicações e outros tipos de solos.
Quadro 3. Teores de mercúrio $(\mathrm{Hg})$, chumbo $(\mathrm{Pb})$, níquel (Ni), cádmio(Cd) e cromo (Cr) em grãos de soja e feijão, em decorrência da aplicação de doses de resíduo de reciclagem de papel e calcário dolomítico

\begin{tabular}{|c|c|c|c|c|c|}
\hline Tratamento & $\mathbf{H g}^{(1)}$ & $\mathbf{P b}^{(1)}$ & $\mathrm{Ni}^{(1)}$ & $\mathbf{C d}^{(1)}$ & $\mathrm{Cr}^{(1)}$ \\
\hline \multirow[t]{2}{*}{$\mathrm{Mg} \mathrm{ha}^{-1}$} & \multicolumn{5}{|c|}{$\mathrm{mg} \mathrm{kg}^{-1}$} \\
\hline & \multicolumn{5}{|c|}{ Soja } \\
\hline 0 & $<0,01$ & $<1,0$ & $1,6 \mathrm{~ns}$ & $<0,10$ & $1,5 \mathrm{~ns}$ \\
\hline 50 & $<0,01$ & $<1,0$ & 1,3 & $<0,10$ & 1,2 \\
\hline 100 & $<0,01$ & $<1,0$ & 1,1 & $<0,10$ & 1,1 \\
\hline 150 & $<0,01$ & $<1,0$ & 0,8 & $<0,10$ & 0,9 \\
\hline 250 & $<0,01$ & $<1,0$ & 0,8 & $<0,10$ & 1,5 \\
\hline 400 & $<0,01$ & $<1,0$ & 0,8 & $<0,10$ & 1,4 \\
\hline 600 & $<0,01$ & $<1,0$ & 0,7 & $<0,10$ & 1,4 \\
\hline \multirow[t]{2}{*}{ Calcário } & $<0,01$ & $<1,0$ & 0,8 & $<0,10$ & 1,9 \\
\hline & \multicolumn{5}{|c|}{ Feijão } \\
\hline 0 & $<0,01$ & $<2,0$ & $1,4 \mathrm{~ns}$ & $<0,10$ & $1,0 \mathrm{~ns}$ \\
\hline 50 & $<0,01$ & $<2,0$ & 1,2 & $<0,10$ & 1,4 \\
\hline 100 & $<0,01$ & $<2,0$ & 1,2 & $<0,10$ & 1,5 \\
\hline 150 & $<0,01$ & $<2,0$ & 1,4 & $<0,10$ & 1,6 \\
\hline 250 & $<0,01$ & $<2,0$ & 0,8 & $<0,10$ & 1,2 \\
\hline 400 & $<0,01$ & $<2,0$ & 0,7 & $<0,10$ & 1,0 \\
\hline 600 & $<0,01$ & $<2,0$ & 1,1 & $<0,10$ & 1,3 \\
\hline Calcário & $<0,01$ & $<2,0$ & 1,0 & $<0,10$ & 1,4 \\
\hline
\end{tabular}

(1) Resultados expressos no material seco a $65{ }^{\circ} \mathrm{C}$. Média de três repetições. Em cada repetição foram feitas duas determinações. ${ }^{n s}$ Diferenças não significativas a $5 \%$.

\section{CONCLUSÕES}

1. O resíduo de reciclagem de papel corrige a acidez e aumenta os teores de $\mathrm{Ca}^{2+}$ em um Cambissolo Háplico muito ácido, bem como incrementa os teores de $\mathrm{P}$ até doses de, aproximadamente, $300 \mathrm{Mg} \mathrm{ha}^{-1} \mathrm{de}$ resíduo úmido.

2. Não há evidências de que o resíduo de reciclagem de papel altera os teores de metais pesados ambientalmente disponíveis no solo e nos grãos de soja e feijão, mesmo em altas doses.

3. As máximas produtividades de grãos de soja e feijão são obtidas nas doses de 323 e $372 \mathrm{Mg} \mathrm{ha}^{-1}$ de resíduo, respectivamente.

\section{AGRADECIMENTOS}

À Companhia Volta Grande de Papel, pelo recurso financeiro para realização deste trabalho. Ao senhor Rodrigo Rocha, por ceder a área experimental. 


\section{LITERATURA CITADA}

ASSOCIAÇÃO BRASILEIRA DE NORMAS TÉCNICAS ABNT. NBR 10004. Resíduos sólidos, classificação de resíduos. Rio de Janeiro, 2004. 63p.

ALMEIDA, J.A.; TORRENT, J. \& BARRÓN, V. Cor de solo, formas do fósforo e adsorção de fosfatos em Latossolos desenvolvidos de basalto do extremo-sul do Brasil. R. Bras. Ci. Solo, 27:985-1002, 2003.

BALBINOT JR., A.A.; TÔRRES, A.N.L.; FONSECA, J.A. \& TEIXEIRA, J. Crescimento e teores de nutrientes em tecido de alface pela aplicação de calcário e resíduos de reciclagem de papel num solo ácido. R. Ci. Agrovet., 5:9$15,2006 \mathrm{a}$.

BALBINOT Jr., A.A.; TÔRRES, A.N.L.; FONSECA, J.A.; TEIXEIRA, J. \& NESI, C.N. Alteração em características químicas de um solo ácido pela aplicação de calcário e resíduos de reciclagem de papel. R. Ci. Agrovet., 5:16-25, 2006b.

BALBINOT Jr., A.A.; VEIGA, M. \& FONSECA, J.A. Aplicação de resíduo de reciclagem de papel em solo ácido: I Fertilidade e teores de metais pesados no solo. Agropec. Catarinense, 23:60-65, 2010a.

BALBINOT Jr., A.A.; VEIGA, M. \& BACKES, R.L. Aplicação de resíduo de reciclagem de papel em solo ácido: II Produtividade das culturas de milho e soja e teores de metais pesados nos grãos. Agropec. Catarinense, 23:66$71,2010 \mathrm{~b}$.

BORGES, M.R. \& COUTINHO, E.L.M. Metais pesados do solo após aplicação de biossólido: II - Disponibilidade. R. Bras. Ci. Solo, 28:557-568, 2004

BORKERT, C.M.; PAVAN, M.A. \& BATAGLIA, O.C. Disponibilidade e avaliação de elementos catiônicos: ferro e manganês. In: FERREIRA, M.E.; CRUZ, M.C.P.; van RAIJ, B. \& ABREU, C.A., orgs. Micronutrientes e elementos tóxicos na agricultura. Jaboticabal, CNPq/ Fapesp/Potafos, 2001. p.151-185.

COMISSÃO SUL-BRASILEIRA DE QUÍMICA E FERTILIDADE DO SOLO - CQFSRS/SC. Manual de adubação e calagem para os estados do Rio Grande do Sul e Santa Catarina. 10.ed. Porto Alegre, 2004. 400p.

CONSELHO NACIONAL DO MEIO AMBIENTE - CONAMA. Resolução $\mathrm{n}^{\circ} 420$, de 28 de dezembro de 2009. "Dispõe sobre critérios e valores orientadores de qualidade do solo quanto à presença de substâncias químicas e estabelece diretrizes para o gerenciamento ambiental de áreas contaminadas por essas substâncias em decorrência de atividades antrópicas.”, Diário Oficial [da República Federativa do Brasil], Brasília, DF, n 249, de 30/12/2009, p.81-84. Disponível em: <http://www.mma.gov.br/port/ conama/legiano1.cfm?codlegitipo $=3 \& a n o=2009>$. Acesso em: mar. 2013.

COSTA, E.R.O.; RIZZI, N.; SILVA, H.D.; SHIZUO, M. \& LAVORANTI, O.J. Alterações químicas do solo após aplicação de biossólidos de fábrica de papel reciclado. Floresta, 39:1-10, 2009.
De MARCO, L.A.; BALBINOT JR., A.A.; OLIVEIRA, T.M.N.; FONSECA, J.A.; COSTA, E.R.O. \& VEIGA, M. Atributos de solo e rendimento da cultura do milho em função da aplicação de resíduo de reciclagem de papel em um Cambissolo Háplico. Agropec. Catarinense, 25:75-79, 2012.

EMPRESA BRASILEIRA DE PESQUISA AGROPECUÁRIA . EMBRAPA. Centro Nacional de Pesquisa de Solos. Solos do Estado de Santa Catarina. Rio de Janeiro, 2004. 1 CD ROM.; mapa color. (Embrapa Solos. Boletim de Pesquisa e Desenvolvimento, 46)

ERNANI, P.R.; NASCIMENTO, J.A.L.; CAMPOS, M.L. \& CAMILLO, R.J. Influência da combinação de fósforo e calcário no rendimento de milho. R. Bras. Ci. Solo, 24:537$544,2000$.

FASSBENDER, H.W. \& BORNEMIZA, E. Química de suelos con enfasis en suelos de America Latina. 2.ed. San José, IICA, 1994. 420p.

FELDKIRCHNER, D.C.; WANG, C.; GOWER, S.T.; KRUGER, E.L. \& FERRIS, J. Effects of nutrient and paper mill biosolids amendments on the growth and nutrient status of hardwood forests. For. Ecol. Manage., 177:95-116, 2003.

GIANELLO, C.; DOMASZAK, S.C.; BORTOLON, L.; KRAY, C.H. \& MARTINS, V. Viabilidade do uso de resíduos da agroindústria coureiro-calçadista no solo. Ci. Rural, 41:242-245, 2011.

GIROTTO, E.; CERETTA. C.A.; SANTOS, D.R.; BRUNETTO, G.; ANDRADE, J.G. \& ZALAMENA, J. Formas de perdas de cobre e fósforo em água de escoamento superficial e percolação em solo sob aplicações sucessivas de dejeto líquido de suínos. Ci. Rural, 40:1948-1954, 2010.

KABATA-PENDIAS, A. \& PENDIAS, H. Trace elements in soils and plants. Bocca Raton, 1984. 315p.

MÄKELA, M.; WATKINS, G.; PÖYKIO, R.; NURMESNIEMI, H. \& DAHL, O. Utilization of steel, pulp and paper industry solid residues in forest soil amendment: Relevant physicochemical properties and heavy metal availability. J. Hazard. Mat., 207-208:21-27, 2012.

MARSCHNER, H. Mineral nutrition of higher plants. London, Academic Press, 1995. 888p.

MEDEIROS, J.C.; ALBUQUERQUE, J.A.; MAFRA, A.L; DALA ROSA, J. \& GATIBONI, L.C. Relação cálcio:magnésio do corretivo da acidez do solo na nutrição e no desenvolvimento inicial de plantas de milho em um Cambissolo Húmico álico. Semina. Ci. Agron., 29:799-806, 2008.

MEDEIROS, J.C.; ALBUQUERQUE, J.A.; MAFRA, A.L.; BATISTELLA, F. \& GRAH, J. Calagem superficial com resíduo alcalino da indústria de papel e celulose em um solo altamente tamponado. R. Bras. Ci. Solo, 33:16571665,2009

NKANA, J.C.V.; TACK, F.M.G. \& VERLOO, M.G. Dynamics of nutrients in tropical acid soils amended with paper pulp sludge. Waste Manage. Res., 17:198-204, 1999.

NOBILE, F.O.; GALBIATTI, J.A.; MUTAISHI, R.I. \& ARAUJO, J.R. Variáveis biométricas da cana-de-açúcar fertilizada com resíduos orgânico e industrial e irrigada com água servida e potável. Eng. Agric., 31:193-200, 2011. 
NOGUEIRA, N.O.; TOMAZ, M.A.; ANDRADE, F.V.; REIS, E.F. \& BRINATE, S.V.B. Influência da aplicação de dois resíduos industriais nas propriedades químicas de dois solos cultivados com café arábica. R. Ci Agron., 43:11-21, 2012.

NOVAIS, R.F. \& SMYTH, T.J. Fósforo em solo e planta em condições tropicais. Viçosa, MG, Universidade Federal de Viçosa, 1999. 399p.

NUNES, J.R.; CABRAL, F. \& LÓPEZ-PINHEIRO, A. Shortterm effects on soil properties and wheat production from secondary paper sludge application on two Mediterranean agricultural soils. Bioresour. Technol., 99:4935-4943, 2008.

OLIVEIRA, H.J.; ERNANI, P.R. \& AMARANTE, C.V. Alteração na composição química das fases sólida e líquida de um solo ácido pela aplicação de calcário e gesso agrícola. R. Ci. Agrovet., 1:93-101, 2002.

PHILLIPS, V.R.; KIRKPATRICK, N.; SCOTFORD, I.M.; WHITE, R.P. \& BURTON, R.G.O. The use of paper-mill sludges on agricultural land. Bioresour. Technol., 60:7380, 1997.
RANGEL, O.J.P.; SILVA, C.A.; BETTIOL, W. \& DYNIA, J.F. Efeito de aplicações de lodos de esgoto sobre os teores de metais pesados em folhas e grãos de milho. R. Bras. Ci. Solo, 30:583-594, 2006.

RÍOS, D.; PÉREZ, C. \& SANDOVAL, M. Phytotoxic effect of paper pulp sludge on Alfisol soil. J. Soil Sci. Plant Nutr., 12:315-327, 2012.

TEDESCO, M.J.; GIANELLO, C.; BISSANI, C.A.; BOHNEN, H. \& VOLKWEISS, S.J. Análise de solo, plantas e outros materiais. 2.ed. Porto Alegre, Universidade Federal do Rio Grande do Sul, 1995. 174p.

UNITED STATES ENVIRONMENTAL PROTECTION AGENCY - USEPA. Final rules: Standards for the use or disposal of sewage sludge. Washington, 1993. Part 503.

VEIGA, M.; PANDOLFO, C.M.; BALBINOT Jr., A.A. \& SPAGNOLLO, E. Chemical attributes of a Hapludox soil after nine years of pig slurry application. Pesq. Agropec. Bras., 47:1766-1773, 2012. 